\title{
INTRODUCCIÓN DE LA MEDIDA DE CALIDAD EN LA PRODUCCIÓN DE AULAS VIRTUALES DE LA UNIVERSIDAD EAN. SEIS SIGMA*
}

\section{QUALITY MEASUREMENT IN PRODUCTION OF VIRTUAL CLASSROOMS AT EAN UNIVERSITY. SIX SIGMA.}

Recibido: 29 de junio de 2018

Evaluado: 2 de septiembre de 2018

Aprobado: 3 de diciembre de 2018

\author{
Henry Manuel Ortega Jiménez ${ }^{*}$ \\ Universidad EAN \\ Orcid: 0000-0002-7963-6233 \\ Luz Carmen Anaya Saladén ${ }^{* \star}$ \\ Universidad EAN \\ Orcid: 0000-0002-7742-1116
Naira Yadira Hernández Rojas ${ }^{\star \star \star \star}$
Universidad EAN
Orcid: 0000-0001-8665-4803

Sandra Rocío Valbuena Castillo ${ }^{\star \star \star \star \star}$

Universidad EAN

Orcid: 0000-0001-7091-5896

Cómo citar este artículo: Ortega Jiménez, H. M., Anaya Saladén, L. C., Hernández Rojas, N. Y. y Valbuena Castillo, S. R. (2019). Introducción de la medida de calidad en la producción de aulas virtuales de la universidad EAN. Seis Sigma. Revista Estrategia Organizacional, 8 (1). pp. 59-83. doi: https://doi.org/10.22490/25392786.3173

* $\quad$ Artículo de investigación. Resultado del Proyecto Seis Sigma realizado en el marco del MBA en la EAN.

** Profesional en Publicidad y Mercadeo, Especialista en Mercadeo y Máster MBA, Universidad EAN. Correo electrónico: ortegajimenez1@hotmail.com.

*** Contadora Pública, Especialista en Administración Financiera, Especialista en Gestión Humana y Máster MBA, Universidad EAN.

**** Ingeniera de Sistemas y Especialista en Gerencia de Proyectos, Universidad EAN.

$\star \star \star \star \star *$ Administradora de Empresas, Especialista Gerencia de Mercadeo, Especialista en Gerencia del Servicio, y Máster MBA, Universidad EAN 


\section{RESUMEN}

La metodología de mejora de la productividad Seis Sigma se ha presentado como una forma efectiva de eliminar y reducir las ineficiencias y defectos en los distintos procesos productivos, aumentando tanto la satisfacción de clientes como la rentabilidad de las compañías. Desde hace más de 25 años, esta metodología ha sido empleada con notable éxito en muchas multinacionales, particularmente, del sector de productos masivos y áreas operativas. Con base en un enfoque reflexivo y de revisión documental sobre los aspectos fundamentales del modelo de Seis Sigma, se plantea la búsqueda de la excelencia en los procesos productivos, se describe su inicio como herramienta de control estadístico de procesos y su evolución como estrategia de gestión de calidad. Además, se abordan los conceptos básicos que hacen viable su integración con la visión y misión de las organizaciones y se promueve la apropiación del modelo DMAMC - definir-medir-analizar-mejorar-controlar- para realizar propuestas de investigación con énfasis en la implementación de Seis Sigma en empresas de servicios de educación superior, contando con el trabajo colectivo integrado de todos los colaboradores en la búsqueda de la mejora continua de procesos. El alcance del presente abordaje del modelo Seis Sigma pretende introducir su metodología en el proceso de Gestión del Conocimiento, específicamente en el área de producción de aulas virtuales de la Universidad EAN.

Palabras clave: Seis Sigma, gestión de calidad, administración, economía.

\section{ABSTRACT}

The productivity improvement methodology called Six Sigma has been presented as an effective way to eliminate and reduce inefficiencies and flaws in the various production processes, increasing both customer satisfaction and profitability of the companies. For 25 years, this methodology has been employed with great success in many multinationals, particularly the mass-market sector. Based on a reflective document review on the fundamentals of the Six Sigma approach, the pursuit of excellence in production processes arises. Its own principle as a tool of statistical process control is described as is its current incorporation into strategy quality management. In addition, the elementary concepts that enable its association with the vision and mission of business are addressed. This article outlines and defines ownership model DMAIC to be used in research with emphasis on the implementation of Six Sigma in service companies' higher education, with the integrated stakeholder's teamwork in the pursuit of continuous process improvement. The 
scope of the present approach of the Six Sigma model aims to introduce its methodology in the Knowledge Management process, specifically in the area of production of virtual classrooms of the EAN University.

Keywords: Six Sigma, Quality Management, Administration. Economy.

\section{INTRODUCCIÓN}

Seis Sigma (6 $\sigma$ ) es un enfoque administrativo con estrategia, táctica y técnica metodológica, que permite detectar objetiva, cuantitativa y cualitativamente dónde, cómo, cuándo y con qué frecuencia se presentan los cuellos de botella de los procesos productivos.

En el presente artículo, cuya finalidad es introducir su método en la producción de aulas virtuales de la Universidad EAN, se aborda el modelo de mejoramiento de la productividad y la calidad denominado Seis Sigma a partir de la metodología de la investigación documental y descriptiva.

La abundante literatura académica y técnica encontrada en la revisión bibliográfica describe los elementos conceptuales y operacionales de este modelo de diseño y mejoramiento de procesos, con base en diagramas, histogramas, análisis de Taguchi, matrices de consolidación y análisis de datos, listas de verificación, ilustraciones de ciclos, herramientas esquemáticas, fórmulas de control estadístico, metodologías de tormentas de ideas que hacen parte de este modelo.

En cuanto al "estado del arte" del que trata de manera importante la investigación documental cuyo propósito no se concibe "como un inventario del conocimiento, sino que implica análisis de la información documental revisada, tomando en cuenta consideraciones y criterios contextualizadores" (Bernal, 2010, p. 112), para introducir el objeto de estudio didácticamente desde los conceptos y fundamentos del modelo Seis Sigma, conviene hacer la analogía de dos obras cinematográficas que apuntan en ese sentido: The LunchBox, una comedia romántica india inspirada en los dabbawalas -los repartidores de comida de Bombay- y Guerra Mundial Z.

En una escena de The LunchBox, la protagonista discute con el mensajero por haberse equivocado con el domicilio, a lo que éste le responde molesto: "iLos de la Universidad de Harvard dicen que nunca cometemos un error! 
Introducción de la medida de calidad en la producción de aulas virtuales de la universidad EAN. Seis Sigma

¿Usted va a ser más lista que los de Harvard?"1. Cuestión que no es ficción. De hecho, en 2010, Harvard Business School indexó el estudio de caso "The Dabbawala System: on-time delivery, every time", como ejemplo de su alto nivel de servicio, equivalente a Seis Sigma o mejor, por su simple sistema operativo con un bajo costo (Thomke y Sinha, 2010-2013).

Por su parte, en la película Guerra Mundial $Z$ una escena del personaje de Brad Pitt le pregunta a un agente de inteligencia israelí cómo fue que previeron algo tan improbable como una invasión zombie, a lo que él responde explicándole la "Regla del Décimo Hombre" tomado de la película-2:

Siempre que nueve miembros del consejo estuvieran de acuerdo en algo de manera unánime, el décimo necesariamente tenía que estar en contra de los otros nueve -aunque, en el fondo, pensara igual que ellos-. Asumir la postura contraria, significaba contradecir la opinión de los nueve restantes.

1 Frase literalmente extractada de la traducción en subtítulos. La película fue estrenada en septiembre de 2014 en salas de cine de Colombia con el título "Los Sabores del Amor".

2 Frase extractada literalmente de la traducción en subtítulos. La película fue estrenada en canales premium de televisión por suscripción en 2014; en salas de cine de Colombia, en junio de 2013. Título original «World War Zw. Ver créditos en Bibliografía.
Dado que los nueve del consejo pensaban que no había tal cosa como una 'epidemia zombie', el décimo asumió que sí la había, y comenzó a investigar sobre la base de esa premisa, permitiendo que Israel construyera defensas, anticipándose al ataque (Gardner, Kleinier, Pitt, Carnahan, Goddard, Lindelof, Straczynski, Brooks y Forster, 2013).

¿Por qué comenzar haciendo referencia a estas escenas de película? Es una forma de conectar el modo didáctico como Eckes (2004), Membrado (2013), Wheat (2008) y Pande junto con Neuman, Cavanagh, (2004, 2002a, 2000) y Holpp (2002b), cada uno a su manera, abordan el modelo Seis Sigma, antes de entrar en la metodología (Escalante, 2013), las bases estadísticas (Gómez, Vilar, Tejero, 2003), las técnicas (Gutiérrez, De la Vara, 2013) y las herramientas de medición (Kumar, 2008), entre otros autores consultados. Tras escuchar ésta suposición, cualquier administrador, contador e ingeniero con especialización en gestión humana, gerencia del servicio, gerencia de mercadeo, gerencia de proyectos o financiera, y con fundamentación de liderazgo en cuadros de mando integral -mas no operativos ni de proceso ni procedimiento estadísticos-, estrategia con resultados y ética empresarial, se preguntaría si dicha teoría es cierta, porque la "Regla del Décimo Hombre" vendría como 
anillo al dedo para asemejarla en la práctica del mejoramiento continuo llamada Seis Sigma.

Cierta analogía con la creativa probabilidad del décimo hombre tiene parangón con la argumentación de Barbara Wheat en el libro "Seis sigma: una parábola sobre el camino hacia la excelencia y una empresa esbelta", sustentada a través de evidencias con lo que concluye: "para que ocurra un cambio es preciso que toda la organización sepa que la manera actual de hacer las cosas no es satisfactoria" (Wheat, 2008, p. 58). Una premisa con la que se indaga acerca del control de defectos, quejas y costos de la desviación estándar de los procesos productivos (sigma).

\section{MARCO TEÓRICO}

Sin entrar aún en las siglas que cada autor o traducción da sobre las metodologías más recurrentes (TQM, EFQM, ISO9001, Lean Manufacturing, Gemba-Kaizen, QFD, DOE, VSM, SMED o Método Taguchi), incluida las cinco eses (5s) para aplicar a partir del lugar de trabajo (Wheat, 2008, p 39) ${ }^{3}$, cabe dilucidar que el ejercicio colectivo por alcanzar un nivel de calidad Seis Sigma confirma que, cuando se investiga la causa de un error de producción o de servicio, o se intenta encontrar la solución

3 "5S" que en inglés son: sifting, sorting, sweeping and washing, standaridizing, self-discipline; pero en español, serían: tamizar- clasificar- barrer/lavar- estandarización-autodisciplina. a un problema, lo mejor es crear un grupo de trabajo multidisciplinario que, con herramientas precisas y la convergencia de diferentes opiniones, se encuentre las mejores respuestas para mejorar los procesos, reducir riesgos, evitar sobrecostos, minimizar desperdicios y aumentar la rentabilidad.

Adicionalmente, es muy importante para el éxito del Modelo Seis Sigma que haya un compromiso genuino por parte de la gerencia de la organización, una filosofía orientada a la excelencia que permee el enfoque de la empresa, los procesos y las mediciones estadísticas (Gutiérrez, 2013), que permita focalizar los esfuerzos hacia el mejoramiento de la calidad, la generación de valor y el incremento de la productividad y de las utilidades.

El modelo Seis Sigma es tanto una estrategia de gestión como una herramienta que permite definir, cuantificar, analizar, corregir y reducir la frecuencia en que se cometen errores en los procesos (Pande, Neuman \& Cavanagh, 2004).

Lo que acerca didácticamente la utilidad del modelo Seis Sigma al caso del "Million Dollar Space Pen"4, en el que se describe que como

$4 \quad$ Parábola moderna de frecuente uso en los coaching motivacionales y de márketing sobre la necesidad de desarrollar la creatividad en el pensamiento estratégico, lo que permite concentrarse en las soluciones, no en los problemas; para que la innovación halle res- 
no se puede escribir en condiciones de cero gravedad con esferos convencionales, la NASA gastó supuestamente -según fuentes extraoficiales - diez millones de dólares en ingeniería avanzada para producir un bolígrafo antigravedad, de tinta presurizada que pudiera escribir en el espacio, bajo el agua, en cualquier ángulo y en un amplio espectro de temperaturas. Sin embargo, luego de diez años de investigación para desarrollar el esfero que escribiera en las condiciones experimentadas durante un viaje espacial, no lo consiguieron. La solución de los rusos ante el mismo problema fue simple: "les dimos un lápiz" (Atkinson, 2012, p. 124). Asimismo, para la confiabilidad de procesos y la confianza de clientes en la gestión de calidad, una solución es el modelo Seis Sigma, como el lápiz para escribir en el espacio sin gravedad (Kumar, 2009). La analogía del esfero espacial sirve para entender que Seis Sigma es una manera de liderar el cambio organizacional, con las soluciones precisas para cada inconveniente (Pande y Holpp, 2002).

\section{ANTECEDENTES GLOBALES DE IMPLEMENTACIÓN}

El modelo Seis Sigma tuvo sus inicios en 1987 en Motorola. Más adelante, en 1995, logró posicionarse gracias al empuje que le dio Jack Welch en General Electric, hasta expandir su éxito en empresas de todo el mundo debido

puestas simples. a los resultados que se lograban con su aplicación. Aunque para algunos, "en realidad empezó en 1964 cuando Joseph Juran escribió el libro Managerial Breakthrough, donde se distingue entre control, que es ausencia de cambio, y avance decisivo, que sí es cambio" (Wheat, 2008, p 52) y para otros, sólo era un heredero ilegítimo de la teoría administrativa de Calidad Total, y como tal sólo iba a durar una década hasta que fuese desplazado por otro modelo con una sigla o un nombre más vendedor (Elderberg, 2003), pues era "TQM con esteroides" (Gómez, 2003; País, 2013, p. 85). El error en los cuestionamientos fue asumir que Seis Sigma era control de calidad más fórmulas estadísticas. Cuestión que en parte lo es, pero, ante la crítica, Mikel Harry, co-creador de Seis Sigma y consultor de Motorola en su primer modelo, apunta:

La antecesora de Six Sigma fue la TQM (Total Quality Management), pero no dio los resultados que prometía por múltiples razones. Para empezar, carecía de un objetivo específico; Six Sigma, en cambio, tiene una meta concreta que consiste en lograr menos de 3,4 defectos por millón de oportunidades. En cierto sentido, más que un sistema de gestión con un fundamento científico; la TQM era una filosofía. Por otra parte, no ponía el foco en las mediciones, mientras que en Six Sigma juegan un papel crítico. De hecho, hasta la retribución 
https://doi.org/10.22490/issn.2539-2786

de los ejecutivos está vinculada con los parámetros de rendimiento. (Alonso, 2003, p. 2).

Las referencias y antecedentes de Seis Sigma descubren una historia con línea de tiempo al que se le atribuye raíces incluso desde el principio cronológico de la teoría administrativa -como efectivamente sucede en prácticamente todas las fuentes consultadas-, de lo cual se infiere que Seis Sigma ha sabido sobrevivir y evolucionar al último grito de la moda, de los modos y de los modelos de calidad.

Por otro lado, es importante mencionar que el modelo Seis Sigma si bien utiliza varias herramientas estadísticas, realmente no se inventó ninguna; sino que combinó los métodos básicos y sacó provecho del papel de la estadística en la administración de los procesos de producción para el mejoramiento de la calidad (Hitoshi, 2002).

Las estrategias Seis Sigma son una evolución de las metodologías de la calidad, más que una revolución de las teorías. En realidad, corresponden a cierto énfasis de las ideas cronológicamente anteriores y a una mejor alineación con el negocio. Es decir, TQM no es que haya fracasado, sino que, por el contrario, creó las bases para el éxito de Seis Sigma.
A manera de las denominaciones de las tecnologías de información, se podría proponer que Seis Sigma está en fase 4G, cuarta generación, siguiendo su transcurso así: la primera generación, cuando pasó la prueba de implementación como enfoque estadístico en procesos productivos masivos, con el ejemplo de éxito en Motorola, una empresa que logró reconvertirse justo a tiempo del comienzo del auge de la revolución tecnológica (Gómez et al, 2004). La segunda generación, cuando demostró ser un sistema eficaz centrado en la gestión y el aumento de utilidades, con el ejemplo dado por Jack Welch en General Electric (Gutiérrez, 2013, p. 399). Como herramienta, sistema y metodología en estas primeras experiencias piloto, surgidas dentro de empresas en busca de la calidad, con base en los conceptos de W. Edward Deming y Joseph Juran (Pande, 2002a, xxiii), y cuyos resultados fueron elocuentes:

Motorola logró 1000 millones de dólares en ahorros durante tres años y el premio a la calidad Malcolm Baldrige en 1988.

Allied Signal, una compañía aeroespacial y de piezas automotrices, ahorró más de 2000 millones de dólares entre 1994 y 1999.

GE alcanzó más de 2570 millones de dólares en ahorros en tres años (1997-1999). (Gutiérrez, p. 398). 
La tercera generación se podría etiquetar cuando se constituye en una estrategia que gravita entre las ideas de coaching -formación de cinturones negros y verdes - y la combinación de técnicas estadísticas, integrándose con otras modalidades, el Lean Manufacturing, por ejemplo, para generar valor real en los clientes (Figuerola, 2009). Como la historia lo documenta Seis Sigma evolucionó de la implementación de su fundamentación metodológica, conformada por sus implementos, herramientas, sistema de procesos y metodologías en torno a la calidad de los productos y las utilidades de la empresa, hasta convertirse en toda una estrategia de gestión de organizaciones, o la cuarta generación, 4G. En suma, la cuarta generación de Seis Sigma, incluye el rendimiento óptimo de la empresa mediante la reducción de defectos en los procesos para la mayor satisfacción del cliente, con foco en el cambio de cultura organizacional.

\section{DEL TQM AL DMAMC}

Si bien se podría considerar Seis Sigma como la TQM actualizada, ampliada y revisada, pues a través de herramientas estadísticas mantiene al día a la empresa en cuanto a estándares, normativas ISO y requisitos de calidad (Barba, Boix, Cuatrecasas, 2000); también hace parte de su enfoque lograr mejoras en la dirección (Chase, 2012), al tiempo que proporciona análisis para razonar sobre soluciones eficaces (Gómez et al, 2003), empleando un modelo de mejora de procesos adaptables (Gómez, 2004) que con su esquemático DMAMC - definir, medir, analizar, mejorar y controlar- permite estructurar paso a paso el seguimiento del qué, el porqué y el cómo de la calidad total.

No en vano, César Alierta Izuel, CEO de Telefónica S.A. y presidente del Consejo Empresarial para la Competitividad de España, plantea que una formación que implique a los empleados es fundamental para guiar el cambio cultural que conlleva una iniciativa de Seis Sigma, pues "la piedra angular de una implantación exitosa es un liderazgo proactivo de la gerencia en todos los procesos" (Pande, 2002a, prólogo, p. xiv). Planteamiento que complementa ampliamente el concepto de Pande:

Seis Sigma es un sistema completo y flexible para conseguir, mantener y maximizar el éxito en los negocios. Seis Sigma funciona especialmente gracias a una comprensión total de las necesidades del cliente, del uso disciplinado del análisis de los hechos y datos, y de la atención constante a la gestión, mejora y reinvención de los procesos empresariales. (Pande, 2002a).

A propósito, según Joseph Moses Juran (19042008), teórico del Management of Quality Control, la calidad se puede definir tanto como 
"aptitud para el uso" (Gómez, 2003, p. 33) como "resultado del diseño, no de la inspección", según William Edwards Deming (1900-1993), difusor de la Calidad Total, (Gómez, 2003, p. 13). De las definiciones de los gurúes de la calidad del siglo XX, surge la estrategia Seis Sigma que no pretende demeritar las teorías de sus predecesores sino fortalecerlas con una visión más integral, con un sólido soporte estadístico y orientada a la productividad y a la generación de valor. No debate sobre los orígenes del significado de calidad cuando se aplica más allá de las funciones de producción o de fabricación (Pande, 2002 a, p. 38-42): dicho de otro modo, cuando se mira en los procesos, no en el producto; o cuando la calidad técnica llega a manos de quien la usa: el cliente y sus valores subjetivos, que a su vez, es quien, en definitiva, da visto bueno al proceso, y como resultado, la marca o empresa gana confianza, el apellido de la calidad.

De hecho, desde la primera generación de Seis Sigma, la satisfacción del cliente fue el objetivo en la innovación de productos tecnológicos y equipos de telecomunicaciones, semiconductores y microprocesadores, cuando apenas comenzaba el desarrollo de nuevas tecnologías, tal cual lo expresa Christopher B. Galvin, CEO de Motorola: "para competir en el creciente mercado internacional re-creamos la forma en que Motorola diseñaba y fabricaba beepers y también otros productos. Fue el comienzo de nuestra iniciativa de Calidad Seis Sigma, y de un nuevo énfasis en la Satisfacción Total del Cliente" (Motorola, 1997, 11':37").

No obstante, en la revisión de los índices analíticos y glosarios de la literatura consultada, no hay evidencia de una nueva definición de calidad tal cual, ya que Seis Sigma como herramienta y estrategia se fija en sus antónimos: la probabilidad de error (Valderrey, 2013) y los indicadores críticos de la calidad (СTQ: Critical to Quality), definidos con gran aporte de, por y para los clientes, alineados y sincronizados con los objetivos del negocio (Escalante, 2013, p. 84). Para ello, introduce términos empleados en metrología y estadística que logran indicadores válidos y fiables en la búsqueda de mejora de procesos, con los que se mide la "voz del cliente" (que viene siendo un indicador "acuraz", ${ }^{5}$ como dice Gómez Dacal, 2004, p. 15), descubriendo las potenciales equivocaciones $y$ puntos críticos de los procesos. Pande lo ejemplifica con un caso verídico:

\footnotetext{
5 Acuraz" no existe en la nueva edición de la Real Academia Española. Es un término que proviene del inglés "accuracy" traducido como grado de corrección, exactitud (de cifras), precisión (en las armas, por ej.), veracidad; fidelidad; lo acertado, lo certero; la exactitud de ajuste.
} 
Sabemos de una imprenta que encauzó a sus equipos hacia la eliminación de milímetros de desviación en el grosor de papel -un importante factor de calidad, seguro-, mientras que se olvidaron de los procesos de seguimiento de pedidos. Aunque la calidad del producto era excelente, los clientes no lo recibían a tiempo. (Pande, 2002a, p. 44).

Es decir, el foco de Seis Sigma está en entender que el conjunto de áreas y actividades de una empresa influye de manera positiva en todos los aspectos de la calidad. En otras palabras, responde qué es la calidad, centrándose en lo que no es. Pero, entonces, ¿qué es mala calidad? Aunque la respuesta parece obvia, el ejercicio está en plantear la respuesta de modo que sean medibles las equivocaciones y fallas de todo tipo en los procesos, por ejemplo: desperdicios y retrasos en la producción, fallas y cuellos de botella en el proceso, PQR, errores en los productos y servicios, inspecciones excesivas, demasiadas instrucciones, presión a los trabajadores, devoluciones de productos, problemas con proveedores, aumento de los servicios de garantía, clientes insatisfechos, retro procesos, pérdidas de ventas, problemas, diferencias y conflictos humanos en el interior de la empresa (Gutiérrez, 2013).

El mínimo común denominador de las situaciones señaladas es que son factores que implican más gastos, menos productos y menos ventas, lo cual se expresa en baja competitividad en precio o en tiempos de entrega, así el producto sea de calidad "certificada". Actualmente, en pleno ascenso del nuevo milenio, es un riesgo que no se puede correr, ya que la globalización es una realidad tan contundente que deja poco lugar a dudas acerca de la necesidad de que las empresas enfrenten rápida y eficazmente la competencia.

Sintetizando, el potencial de Seis Sigma en lo que se puede considerar como su cuarta generación está en su flexibilidad (Pande, 2004) y funcionalidad como estrategia de gestión planificada (Kumar, 2009), que se centra en los resultados de calidad (Schroeder, Meyer Golstein, Rungtusanatham, 2011) y en el "retorno de la inversión" (Vitasek, Manrodt, Kling, 2013), con el objetivo de promover cambios significativos en las organizaciones (Barba, 2000), siempre en busca de mejoras en los procesos (Escalante, 2013), productos y servicios ofrecidos a los clientes. (Eckes, 2004) (Ventura, 2014).

\section{CÓMO SE MIDE Y QUÉ MIDE SEIS SIGMA $(6 \Sigma)$}

Según Jack Welch, ex presidente de General Electric, elegido Ejecutivo del Siglo en 1999 por la revista Fortune, "el corazón de Six Sigma suscita una idea que podría poner una empresa 
al revés, cambiando el enfoque de la organización. La visión sin acción es un sueño. Acción sin visión es simplemente pasar el tiempo. Acción con visión es hacer una diferencia positiva. Cuando el ritmo de cambios dentro de la empresa es superado por el ritmo de cambios fuera de ella, el final está cerca" (Wobi, 2013).

El término original de Seis Sigma proviene del símbolo " $\sigma$ " ("sigma") que significa una medida de la variabilidad de la calidad o desviación estándar, utilizada para indicar qué parte de los datos presentados en el proceso productivo falla. Cuanto menor sea la desviación estándar del proceso mayor será la calidad de los productos o servicios producidos. En términos del modelo Seis Sigma entre más veces quepa la desviación estándar entre el límite permitido superior y el límite permitido inferior de un proceso, mayor será la calidad del proceso, debido a que los niveles de variabilidad del proceso están controlados y son muy bajos.

La idea fundamental es encontrar las causas raíz de los defectos para que a partir de esta información se mejoren y se controlen los procesos y de esta manera se pueda reducir la ocurrencia de defectos a niveles tan bajos como 3,4 veces por millón de oportunidades.
Tabla 1. Nivel de calidad de Seis Sigma.

\begin{tabular}{|c|c|c|}
\hline \multicolumn{3}{|c|}{ Como calcular el nivel de calidad sigma } \\
\hline Nivel en Sigma & DPMO & Rendimiento \\
\hline 6 & 3.40 & $99.9997 \%$ \\
\hline 5 & 233.00 & $99.98 \%$ \\
\hline 4 & 6.210 .00 & $99.3 \%$ \\
\hline 3 & 66.807 .00 & $69.15 \%$ \\
\hline 2 & 308.537 .00 & $30.85 \%$ \\
\hline 1 & 690.000 .00 & $99.9997 \%$ \\
\hline 0 & 933.200 .00 & $6.68 \%$ \\
\hline
\end{tabular}

Fuente: elaboración propia

Desde la primera generación de Seis Sigma el defecto fue medido con base en una métrica, no como algo abstracto. Como se parte de entender que todo proceso tiene defectos, por ende, bajar la tasa de éstos, es lo buscado con el modelo Seis Sigma, un proceso de mala calidad es errático, costoso, inestable y no se puede predecir. En definitiva, la mala calidad es inversamente proporcional a la competitividad (Gutiérrez, et al, 2013).

La fortaleza del Seis Sigma está en que es una metodología que hace más sencilla la formulación del problema en los procesos y la identificación de las soluciones. Es decir, facilita encontrar una métrica del problema o de la falla que hace más fácil expresarlo, entenderlo y controlarlo. 
En la tabla 1 se presenta una manera de plantear y cuantificar problemas. En la primera columna se indica que evitar, en la segunda columna se describe un problema a solucionar y en la última columna se observa la métrica con la cual se debe tratar el problema.

Tabla 2. Planteamiento y cuantificación de problemas.

\begin{tabular}{|l|l|l|}
\hline \multicolumn{1}{|c|}{ Evitar } & \multicolumn{1}{|c|}{ No } & \multicolumn{1}{c|}{ Sí } \\
\hline Preguntas & $\begin{array}{l}\text { ¿Cómo podremos reducir el tiempo } \\
\text { muerto en la línea de ensamble? }\end{array}$ & $\begin{array}{l}\text { El tiempo muerto en la línea de ensamble } \\
\text { actualmente es del 35\% de las horas } \\
\text { de operación. }\end{array}$ \\
\hline La palabra "falta" & Falta un software actualizado. & $\begin{array}{l}\text { La información para subir al sistema tiene un } \\
\text { retraso de cinco días. }\end{array}$ \\
\hline $\begin{array}{l}\text { Enmascarar una solución } \\
\text { como problema }\end{array}$ & $\begin{array}{l}\text { Necesitamos contratar otro encargado } \\
\text { de embarque para el almacén }\end{array}$ & $\begin{array}{l}\text { El 30\% de los embarques programados no se } \\
\text { están liberando a tiempo. }\end{array}$ \\
\hline $\begin{array}{l}\text { Culpar a la gente en lugar } \\
\text { de los procesos }\end{array}$ & $\begin{array}{l}\text { Los archivistas no están haciendo } \\
\text { su trabajo }\end{array}$ & $\begin{array}{l}\text { No se pueden localizar archivos dentro } \\
\text { de los cinco minutos permitidos después de } \\
\text { haberlos solicitado. }\end{array}$ \\
\hline
\end{tabular}

Fuente: elaboración propia a partir de Ventura (2014)

En el modelo Seis Sigma entre más veces quepa la desviación estándar entre el límite permitido superior y el límite permitido inferior de un proceso, mayor será la calidad del proceso, debido a que los niveles de variabilidad del proceso están controladosy son muy bajos, o muy cercanos a 3.4 defectos en un millón de oportunidades (Gutiérrez, 2013).

Para ponerlo de manera práctica relacionamos en la tabla 2 el número de defectos en un proceso cualquiera con su correspondiente número de Sigmas, combinando las tablas de conversión en Gómez (2004, p. 22) y Pande (2002a, p. 25):

Tabla 3. Niveles Sigma y rendimiento.

\begin{tabular}{|c|l|l|l|}
\hline Nivel sigma & \multicolumn{1}{|c|}{$\begin{array}{c}\text { Defectos por millón de } \\
\text { oportunidades }\end{array}$} & $\begin{array}{c}\text { Expresado } \\
\text { en \% de errores }\end{array}$ & \multicolumn{1}{|c|}{ Rendimiento } \\
\hline 2 & 308.537 & $30,8537 \%$ & $69,2 \%$ \\
\hline 3 & 66.807 & $6,6807 \%$ & $93,3 \%$ \\
\hline 4 & 6.210 & $0,621 \%$ & $99,4 \%$ \\
\hline 5 & 233 & $0,0233 \%$ & $99,98 \%$ \\
\hline 6 & 3.4 & $0,00034 \%$ & $99,99966 \%$ \\
\hline
\end{tabular}

Fuente: Gómez (2004, p. 22) y Pande (2002a, p. 25) 
La manera más efectiva de apropiar los niveles Sigma se encuentra en la realidad de la globalización económica: un porcentaje de 6,6807 \% de imperfectos implica 66.807 artículos defectuosos por cada millón (PPM) producido. En un mundo donde las cifras de consumo anual para muchos productos son de varios millones, esa cantidad de imperfecciones es intolerable. Por ejemplo, si Apple fabricara 10 millones de Iphone6 con calidad Tres Sigma, esto implicaría que 668.070 consumidores tendrían problemas con su nuevo modelo de celular. Esa cifra acabaría con el prestigio global y la confianza mundial hacia la marca, así 93,3\% de sus usuarios fieles obtuvieran el celular con mayor innovación de todos.
Igualmente, si por cada millón de servicios haya 66.807 errores es un lujo que en el contexto de la competitividad global una empresa no se puede dar, bien sea en envíos que no lleguen a tiempo, para una compañía de mensajería; clientes insatisfechos en menos de un año para una cadena de almacenes; en quejas de pasajeros para una línea aérea; o, peor aún, en medicamentos defectuosos para una empresa farmacéutica. Una cifra que es completamente inaceptable en procesos que afectan la salud o la seguridad de las personas. En resumen, la calidad de Tres Sigma implica demasiados errores. En la práctica, con el siguiente recuadro queda más patentado:

Tabla 4. Comparación $3 \sigma$ vs 6 o, en la práctica.

\begin{tabular}{|c|c|}
\hline Calidad Tres Sigma igual a & Calidad Seis Sigma igual a \\
\hline $\begin{array}{l}\text { Por lo menos } 54.000 \text { prescripciones } \\
\text { médicas erradas por año }\end{array}$ & Una prescripción médica errada en 25 años. \\
\hline $\begin{array}{l}27 \text { minutos fuera del aire por canal } \\
\text { de TV cada semana }\end{array}$ & $\begin{array}{l}2 \text { segundos fuera del aire por canal } \\
\text { de TV cada semana }\end{array}$ \\
\hline 1,35 palabras mal escritas por página por libro & 1 palabra mal escrita en diez enciclopedias \\
\hline 20.000 artículos de correo perdidos por hora & Siete artículos perdidos por hora. \\
\hline $\begin{array}{l}\text { Tomar agua insalubre por cada } \\
15 \text { minutos diariamente }\end{array}$ & Un minuto inseguro cada siete meses \\
\hline $\begin{array}{l}5.000 \text { intervenciones quirúrgicas incorrectas } \\
\text { a la semana }\end{array}$ & 1,7 operaciones incorrectas por semana \\
\hline Falta de electricidad por casi siete horas al mes & Una hora sin electricidad cada 34 años. \\
\hline $\begin{array}{l}698 \text { aterrizajes forzosos en un gran aeropuerto } \\
\text { internacional cada mes }\end{array}$ & $\begin{array}{l}1 \text { aterrizaje forzoso en un aeropuerto } \\
\text { internacional cada mes. }\end{array}$ \\
\hline
\end{tabular}

Fuente: elaboración propia a partir de tablas de Barbosa (2013, pp 194-195), Escalante (2013, p. 19) y Membrado (2013, p. 136) 
En conclusión, la finalidad de Seis Sigma es mejorar los procesos para que los productos generen satisfacción a los clientes. En la práctica, aunque no todos los procesos de una empresa logren nivel de calidad Seis Sigma, tener ese propósito significa diseñar procesos en los que la variación de las características de calidad sea tan pequeña que sus especificaciones en términos prácticos equivalen a un proceso con defectos marginales. (Membrado, 2013). El foco está en entender que la calidad en todas las áreas y actividades influye de manera positiva en todos los aspectos. (Gutiérrez, 2013, pp 389-398).

\section{METODOLOGÍA}

A lo largo de la presente investigación descriptiva y documental sobre el tema, se ha abordado metodológicamente el procedimiento científico de revisión de fuentes con el respectivo proceso sistemático de indagación, recolección, organización, análisis e interpretación de información y datos en torno a Seis Sigma. De acuerdo con las premisas de Bernal (2010, p. 111), se ha comprendido la descripción, registro, análisis e interpretación de la naturaleza actual y la compresión de procesos y fenómenos del Seis Sigma con base en la "información escrita con el propósito de establecer relaciones, diferencias, etapas, posturas y estado actual del conocimiento respecto al tema objeto de estudio".

\section{METODOLOGÍA DE SEIS SIGMA}

La metodología de Seis Sigma está compuesta por cinco etapas que componen la sigla DMAMC -definir-medir-analizar-mejorar-controlar-, que en gran medida son una "modernización" del ciclo PDCA o Rueda de Deming, inspirado a su vez en los Catorce Puntos de Deming, que definía la calidad como "resultado del diseño y no de la inspección" y cuyos puntos tenían como propósito "hacer que la gente disfrute su trabajo" (Gómez, pp. 33-35). En Deming el acrónimo hace referencia a una secuencia basada en Plan-Do-Check-Act (Escalante, 2013, pp 1931), que no ve la mejora de la calidad como algo que tiene un principio y un final determinados, sino que "una vez que finalizamos un ciclo PDCA, el proceso de mejora empieza otra vez" (Gómez, pp 36-38). Como precursor de Seis Sigma, el PDCA también se conoce en español como ciclo PHVA, donde "Plan" determina las directrices para el desempeño corporativo de los procesos según la formulación estratégica; "Do" o "Hacer", es ejecución; "Check" es "Verificación"; y "Act" es la actuación o acciones de mejora y retroalimentación de los procesos de planeación (Ríos, 2013, p. 21).

En el ciclo de Seis Sigma los objetivos se establecen de manera consistente con las demandas del cliente y la estrategia de la empresa (Pande, 2002). DMAMC se centra en la mejora 
de productos, servicios y procesos de los proyectos empresariales existentes (Pande, 2000).

En el arranque del proceso, se requiere por lo tanto fijar con claridad y exactitud el enunciando del problema y las propiedades con las que se designa el objetivo, con lo cual se ayuda a desarrollar las prioridades del negocio relacionándolas con un parámetro -características críticas para la calidad, CTQ-6 importante para el cliente, donde los requisitos críticos pueden ser satisfacción, costo, servicio, entrega o alguna característica importante para el consumidor o usuario (Escalante, pp. 22-90).

Tanto el problema como el objetivo deben ser entendibles para la empresa, de alcance razonable, común a los miembros del equipo de trabajo; además de contar con el apoyo y aprobación ejecutiva. La preparación para ser un eficaz equipo de gestión de proyectos de mejoramiento continuo cuenta con reglas básicas y pautas del grupo, cuyo impacto financiero debe ser validado por el área de finanzas de la empresa (Gómez, 2003, p. 94).

6 Los parámetros de los requisitos críticos o necesidades importantes de los clientes son denominado CTQ, por "Critical-to-Quality" o característica crítica para la calidad, CCC. Para claridad sobre los términos recurrentes, remitirse al Glosario incluido al final del artículo.
Las herramientas y técnicas utilizadas para la etapa de definición podrían incluir gráficas de impacto-desempeño; matriz de selección de proyectos, diagrama de Pareto (flujo); Cuadro de Mando Integral (Balanced Scorecard); mapa de procesos.

En cuanto a la integración de equipos de trabajo en la implementación de Seis Sigma, la creatividad de los miembros del grupo de un proyecto DMAMC es indispensable para conseguir las metas de mejorar tanto la eficacia como la eficiencia. Definir de forma cuantitativa las necesidades de los clientes y lo que constituye un defecto, permite establecer los objetivos de mejora, teniendo en cuenta que "lo importante es lo que quiere el cliente", a la vez que "define claramente cómo los ejecutivos lo usan como su estrategia de administración del proceso empresarial que les ayuda a alcanzar los objetivos del negocio", según Eckes (2004, p. 157), en contra de la idea de beneficios hacia dentro del negocio, en la cual se enfoca la solución de los problemas en las deficiencias de los recursos humanos. O sea, la meta del Seis Sigma parte de la gestión humana con la finalidad de mejorar tanto la eficacia como la eficiencia:

Uno de los problemas de un método de calidad de hace unos años atrás, llamado Reingeniería de procesos, era que virtual- 
Introducción de la medida de calidad en la producción de aulas virtuales de la universidad EAN. Seis Sigma

mente todos los beneficios que resultaban para la administración eran reducciones del personal de trabajo. Cuando los esfuerzos se enfocan exclusivamente en la eficiencia como la reingeniería de procesos- aparecen a menudo como reducciones del personal. Cuando los esfuerzos como el Seis Sigma funcionan bien en cuanto al mejoramiento de la eficacia -el grado en el cual se satisfacen las necesidades y requisitos del cliente-, es típico que el negocio crezca y se amplíe, no que se reduzca. (Eckes, 2004, pp. 155-156).

- Seis Sigma en trabajo de equipo Para lograr que la estrategia Seis Sigma sea beneficiosa es necesario que la empresa sepa exactamente cómo planificar e implementar DMAMC. Por tanto, lo importante es que los empleados estén debidamente capacitados, a través de coaching de liderazgo y entrenamiento en los niveles sugeridos por Seis Sigma, como una labor de equipo (Pande, 2004). De ahí salen las categorías de cinturones que representan el nivel gradual de práctica y constancia de los empleados entrenados en el desarrollo de Seis Sigma. Se entiende que todos los participantes deben colaborar en la medida de sus tiempos y capacidades, para ello Seis Sigma contempla una serie de roles recomendados para poder administrar los proyectos de forma adecuada:
Campeón: es el líder de la empresa, encargado de fijar metas y seleccionar proyectos. Está completamente entrenado y facilita la planeación de los factores estratégicos y del equipo, gestionando recursos para el proyecto. Maestro de Cinturón Negro: apoya en la definición y selección de proyectos. Es el gurú de Seis Sigma y orienta sobre la implementación o herramientas específicas que se requieren en las etapas de mejoramiento y control. Cinturón Negro: es el líder de los proyectos, entrena a los miembros de equipo y está capacitados ampliamente en Seis Sigma. La idea de hacer un paralelo entre las artes marciales y la implantación del Seis Sigma surgió porque ambas dependen tanto de la fuerza, la velocidad y la determinación, como de la disciplina mental y el entrenamiento sistemático e intensivo. Esto quiere decir que tiene aptitudes que le permiten cuantificar los beneficios producidos por un proyecto, así como la habilidad en el manejo e informes de proyectos, y dominio de herramientas estadísticas requeridas para medir y analizar los procesos. Cinturón Verde: colaborador con entrenamiento Seis Sigma que da soporte a los equipos y acelera el despliegue del proyecto, capaz de liderar grupos focalizados que identifican los defectos a través de conocimiento operativo del proceso. (Gutiérrez, 2013). 


\section{PROPUESTA Y VALOR AGREGADO}

\section{MÉTODO DE SEIS SIGMA EN EL PROCESO DE GESTIÓN DEL CONOCIMIENTO: ÁREA DE PRODUCCIÓN DE AULAS VIRTUALES.}

La dirección de gestión del conocimiento es el proceso encargado del diseño, producción, provisión de medios y mediaciones para la prestación de servicios educativos internos y externos desde la generación de contenidos hasta el momento en que se emplean.

Para esbozar una propuesta de estrategia Seis Sigma desde y hacia gestión del conocimiento, se parte de los objetivos de la Universidad EAN y se sintoniza con todas las líneas de trabajo para obtener una perspectiva respecto a los requerimientos de clientes y usuarios.

\section{DISEÑO METODOLÓGICO DE LA PROPUESTA}

La muestra se refiere a las aulas que produce el área de aulas virtuales para la Facultad de Estudios en Ambientes Virtuales en los programas de pregrado. En promedio se producen 150 aulas por ciclo para la Facultad de Estudios en Ambientes Virtuales para los programas de pregrado, las cuales deben estar disponibles 15 días antes de iniciar las actividades académicas, en cuanto a la guía de estudio y el aula virtual, como parte del material de aprendizaje.
La población estuvo compuesta por 44 encuestas realizadas en las aulas de los estudiantes, con el objeto de conocer la opinión de las aulas virtuales como insumo para su desarrollo académico.

El tamaño de la muestra se obtuvo considerando un nivel de satisfacción de los estudiantes siendo el número 1 correspondiente al menor valor y 5 al mayor valor.

\section{DESARROLLO DE LA METODOLOGÍA}

\section{SEIS SIGMA}

Teniendo en cuenta que al aplicar la metodología Seis Sigma la cual permite mejorar la calidad en los procesos disminuyendo errores en las actividades que son causa de bajo nivel de satisfacción del cliente, se aplicó la metodología DMAMC -definir-medir-analizar-mejorar-controlar- en el proceso de gestión del conocimiento, subproceso producción de aulas virtuales, con el fin de disminuir el nivel de clientes insatisfechos y deserción académica por esta causa.

\section{- Definir}

"Definir", por supuesto, significa: "establecer las causas de un problema y acordar sus límites. Esta fase ayuda al equipo a imaginar el proceso en el tiempo y le otorga perspicacia sobre dónde deben estar concentrados los esfuerzos de mejoría, y se aplica a las necesidades y requisitos de los clientes." (Kumar, 2008, pp. 67-69). 
Durante el primer ciclo se encontró un nivel de insatisfacción de los clientes del 66\%, este resultado se obtuvo del análisis de los datos realizado para las aulas en la que los encuestados calificaron con valores 1 y 2 de las variables -oportunidad de los datos, calidad de los contenidos, diseño y funcionalidad-.

A partir de los resultados obtenidos se vio la necesidad de verificar y optimizar el proceso de producción de aulas virtuales para tomar medidas preventivas y correctivas y aumentar el nivel de satisfacción de los clientes. Los estudiantes esperan la activación oportuna de las unidades de estudio ofertadas para cada ciclo en los tiempos establecidos por la facultad y que las aulas virtuales cumplan con la calidad esperada por los estudiantes. Desde la recepción de materiales para la producción de las aulas virtuales hasta la creación y puesta en marcha se realiza en cinco etapas:

Tabla 4. Variables.

\begin{tabular}{|c|c|}
\hline Procesos & Variables a controlar \\
\hline $\begin{array}{l}\text { Recepción del material para ambientes } \\
\text { de aprendizaje y aulas virtuales }\end{array}$ & $\begin{array}{l}\text { Oportunidad en el envío de contenidos } \\
\text { Verificación del cumplimiento de normas sobre } \\
\text { derechos de autor establecidos } \\
\text { Asignación oportuna a Pedagogos }\end{array}$ \\
\hline Adecuación Pedagógica & $\begin{array}{l}\text { Validación de los contenidos por unidad de estudio } \\
\text { Revisión de estilo } \\
\text { Coherencia entre el propósito, instrucciones y producto }\end{array}$ \\
\hline Diseño Ambiente & $\begin{array}{l}\text { Distribución de los materiales para diseño } \\
\text { Ayudas Audiovisuales } \\
\text { Disponibilidad de herramientas para diseño } \\
\text { Requerimiento de Contenido } \\
\text { Conceptualización de lo que se quiere mostrar }\end{array}$ \\
\hline Administración de Aulas & $\begin{array}{l}\text { Recepción oportuna de calendarios por la facultad } \\
\text { Programación de la actividad en aulas virtuales } \\
\text { Oportunidad en la Aprobación del aula virtual del } \\
\text { coordinador } \\
\text { Monitoreo de la integridad del aula }\end{array}$ \\
\hline $\begin{array}{l}\text { Creación y Puesta en Marcha } \\
\text { de Aulas Virtuales }\end{array}$ & $\begin{array}{l}\text { Sistema de Información Académico - SUGAR } \\
\text { Publicación del aula para los usuarios } \\
\text { Registro de Usuarios en el aula virtual }\end{array}$ \\
\hline
\end{tabular}

Fuente: elaboración propia a partir de datos del proceso de gestión del conocimiento - aulas virtuales 


\section{- Medir}

De acuerdo con los datos obtenidos en la producción y activación de aulas virtuales se encontró fallas en 29 productos en los que se identifican cuatro variables a controlar.

Tabla 5. Variables a controlar

\begin{tabular}{|c|c|}
\hline \multirow{2}{*}{ Oportunidad de los datos } & $\begin{array}{l}\text { Se le informó oportunamente su usuario } \\
\text { y contraseña. }\end{array}$ \\
\hline & $\begin{array}{l}\text { Se le habilitaron oportunamente las aulas } \\
\text { de las unidades de estudio }\end{array}$ \\
\hline \multirow{3}{*}{ Calidad de los contenidos } & $\begin{array}{l}\text { Los contenidos son de utilidad para el } \\
\text { desarrollo del bloque temático, módulo o } \\
\text { unidad de estudio }\end{array}$ \\
\hline & $\begin{array}{l}\text { Las lecturas (en sus diferentes formatos) son } \\
\text { utilidad para el desarrollo del bloque temático, } \\
\text { módulo o unidad de estudio }\end{array}$ \\
\hline & Los gráficos y las imágenes son pertinentes. \\
\hline \multirow{3}{*}{ Diseño } & $\begin{array}{l}\text { Las ayudas y recursos permanentes } \\
\text { dispuestos en el aula son de utilidad }\end{array}$ \\
\hline & $\begin{array}{l}\text { Los temas establecidos para los foros } \\
\text { generaron interés. }\end{array}$ \\
\hline & $\begin{array}{l}\text { La presentación de los contenidos del aula } \\
\text { es amigable y atractiva. }\end{array}$ \\
\hline \multirow{2}{*}{ Funcionalidad } & $\begin{array}{l}\text { En el calendario del aula se incluyó la } \\
\text { programación de las distintas actividades }\end{array}$ \\
\hline & $\begin{array}{l}\text { El periodo establecido para el desarrollo de los } \\
\text { foros fue adecuado. }\end{array}$ \\
\hline
\end{tabular}

Fuente: elaboración propia a partir de datos del proceso de gestión del conocimiento - aulas virtuales

\section{- Análisis}

Para analizar detalladamente el proceso de y puesta en marcha de las aulas virtuales que producción de aulas virtuales se realizó un diagrama causa efecto de los factores que pueden incidir en las fallas encontradas en la creación afectan el nivel de satisfacción del cliente en los diferentes procesos. 


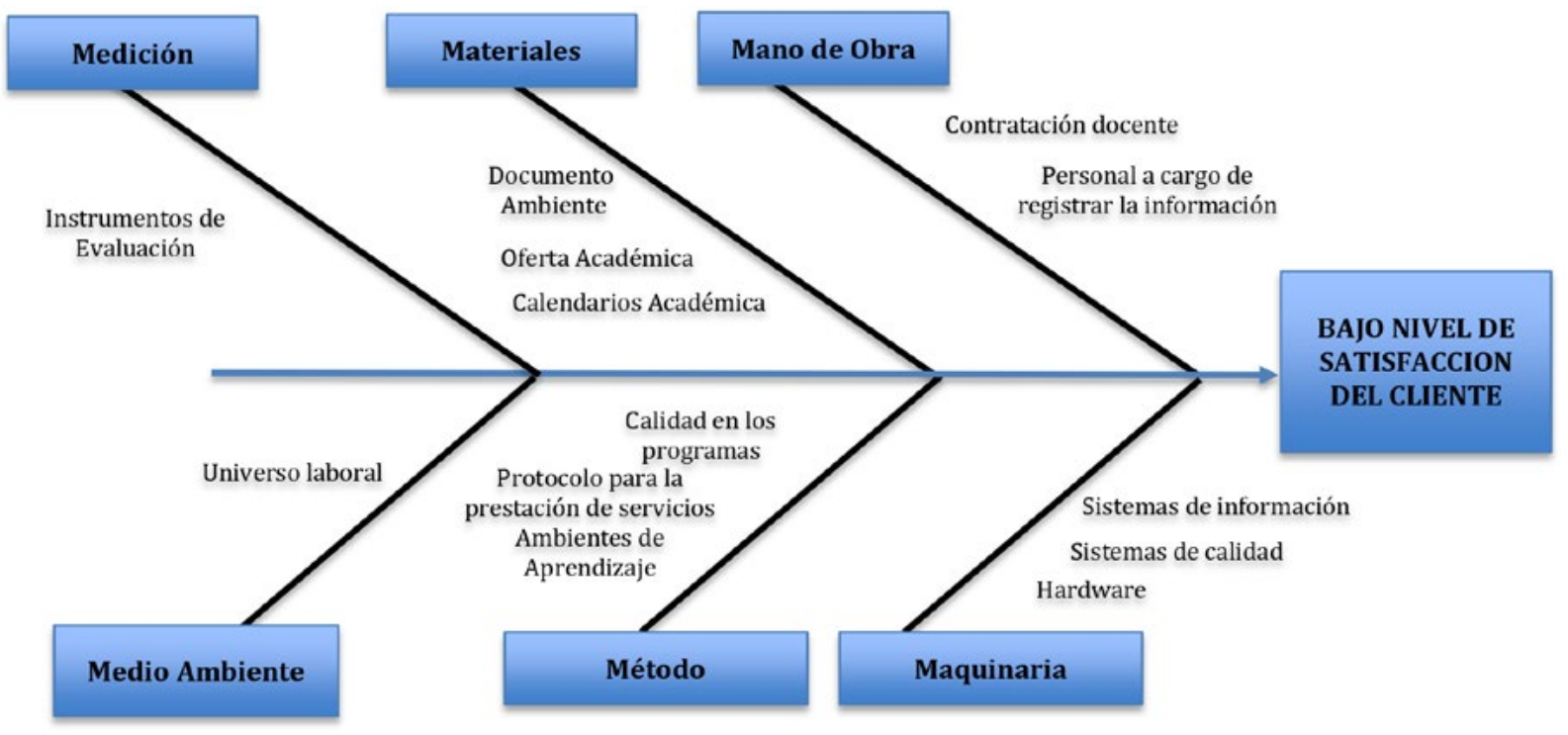

Figura 1. Diagrama de causa efecto de los factores que posiblemente afectan el servicio.

Fuente: elaboración propia a partir de datos de aulas virtuales

Con los datos obtenidos por la voz del cliente a través de la encuesta en la que nos permitió medir la calidad de las aulas virtuales y la oportunidad en la activación de los estudiantes en las mismas, muestra que no hay una distribución normal de los datos como se ve en la figura 1 .

La relación entre el nivel sigma y el total de unidades fabricadas al aplicar la fórmula de DPU (Defects Per Unit).

$$
d p u=\frac{\text { número de defectos encontrados }}{\text { Unidades inspeccionadas }}
$$

Para este caso se tomaron los siguientes datos que se reemplazan en la fórmula:
Tabla 6. Datos

\begin{tabular}{|l|l|}
\hline No. Fallas & 29 \\
\hline Unidades Inspeccionadas & 44 \\
\hline Unidad a Medir & Aula Virtual \\
\hline
\end{tabular}

Fuente: elaboración propia

$$
\begin{gathered}
d p u=\frac{29}{44}=0,65909091 \text { errores } / \text { aula } \\
=0,65909 * 1.000=659 \text { errores } / \text { millón aulas }
\end{gathered}
$$

Si en el ciclo se detectaron 29 errores en las aulas, las oportunidades de error por cada aula virtual son de 3. De acuerdo con la tabla de nivel de calidad de sigma el estado actual de producción de aulas está en un nivel de rendimiento del $93.3 \%$. 


\section{Nivel de Satisfacción del Cliente en Aulas Virtuales}

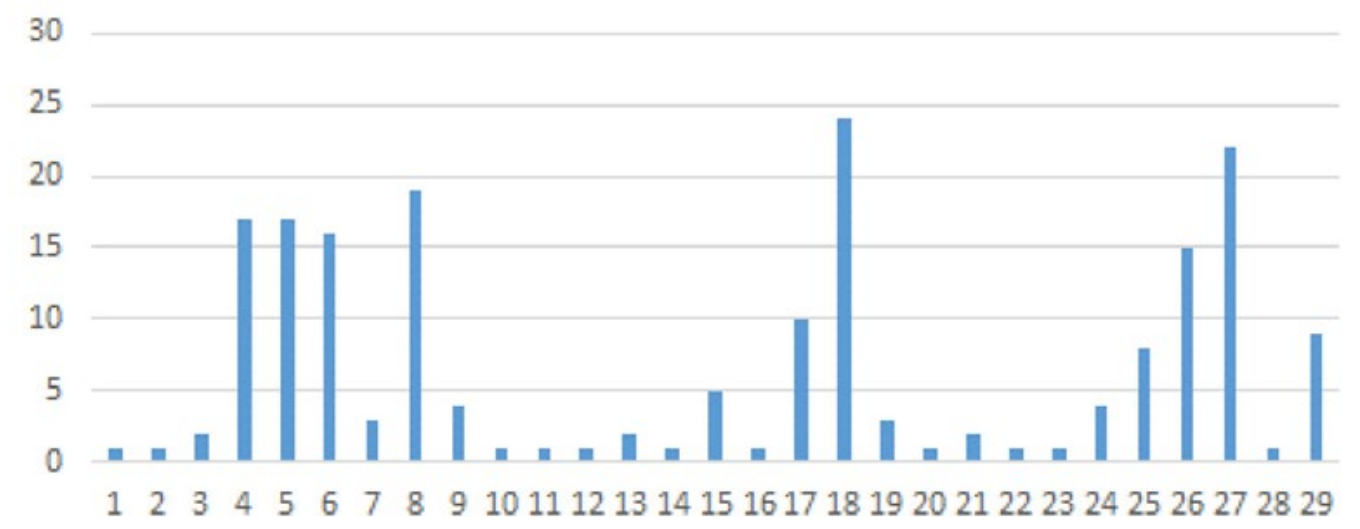

Figura 2. Nivel de satisfacción del cliente de aulas virtuales.

Fuente: elaboración propia a partir de datos del proceso de gestión del conocimiento

Teniendo en cuenta que la serie de datos no muestran una distribución normal se utilizó el programa Minitab para normalizar los datos y encontrar el nivel de tolerancia adecuado, ir evaluando las fallas, determinar sus causas y mejorar el proceso. A continuación se muestra la tabla de datos original y los datos normalizados.

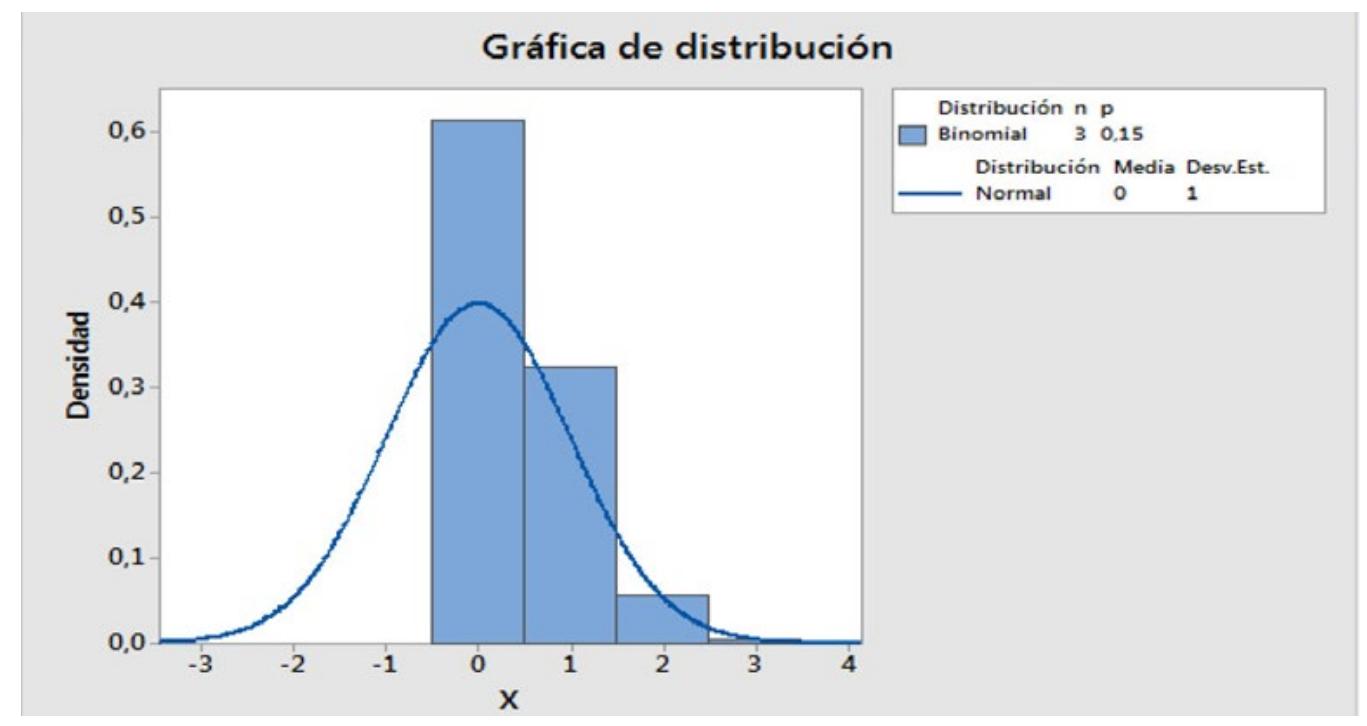

Figura 3. Grafica de distribución.

Fuente: elaboración propia 
Se utilizó estadística descriptiva para normalizar los datos y hallar la desviación estándar y la media.

Tabla 8. Normalización de datos.

\begin{tabular}{|l|l|}
\hline No. Fallas & 29 \\
\hline Media & 0,186 \\
\hline Desviación estándar & 1,000 \\
\hline
\end{tabular}

Fuente: elaboración propia

Se hallaron los límites de tolerancia lo que muestra que en el estado actual del proceso se tiene una desviación de 3 lo cual está por encima de los límites superior e inferior, con un nivel de tolerancia del $95 \%$.

Tabla 9. Límites.

\begin{tabular}{|l|l|}
\hline Limite Superior & 2,755 \\
\hline Límite inferior & -2.383 \\
\hline
\end{tabular}

Fuente: elaboración propia

\section{- Mejorar}

Con el análisis anterior se pueden optimizar las siguientes variables, las cuales se evalúan según la importancia de las variables, siendo alto el nivel más importante y bajo el menos impactante.

Tabla 10. Variables y evaluación.

\begin{tabular}{|c|c|c|}
\hline Variables & Mejora & Evaluación \\
\hline \multirow{2}{*}{$\begin{array}{l}\text { Oportunidad } \\
\text { de los datos }\end{array}$} & $\begin{array}{l}\text { Trabajo en equipo con Tic’s con el fin de entregar las credenciales de ingreso } \\
\text { inmediatamente la matricula se refleje en los sistemas de información. }\end{array}$ & Alto \\
\hline & $\begin{array}{c}\text { Actualización a diario la información de los matriculados para garantizar la } \\
\text { oportuna vinculación de los estudiantes a las unidades de estudio. }\end{array}$ & Alto \\
\hline \multirow{3}{*}{$\begin{array}{l}\text { Calidad } \\
\text { de los contenidos }\end{array}$} & $\begin{array}{l}\text { Los docentes hagan revisión, actualización e innovación de los contenidos de los } \\
\text { módulos, garantizando el enfoque con el tema de la unidad de estudio. }\end{array}$ & Alto \\
\hline & $\begin{array}{l}\text { Los docentes propongan y relacionen bibliografías más amplias sobre los temas } \\
\qquad \text { a tratar, que sean actuales e innovadoras y de fácil acceso. }\end{array}$ & Alto \\
\hline & $\begin{array}{l}\text { Creación en conjunto con la parte de diseño y el docente objetos novedosos, } \\
\text { Ilamativos e innovadores que generen motivación para consulta e investigación. }\end{array}$ & Alto \\
\hline \multirow{3}{*}{ Diseño } & $\begin{array}{l}\text { Revisar, definir y establecer los recursos que son más importantes y útiles } \\
\text { para el los estudiantes. }\end{array}$ & Medio \\
\hline & $\begin{array}{l}\text { Fortalecer los temas de los foros donde se dé más debate entre todos } \\
\qquad \text { los participantes. }\end{array}$ & Medio \\
\hline & Innovar con nuevas tecnologías que se integren a la plataforma Blackboard. & Medio \\
\hline Funcionalidad & Generar alertas de fácil visualización, permitiendo mejor navegación. & Bajo \\
\hline
\end{tabular}

Fuente: elaboración propia a partir de datos de aulas virtuales 
- Controlar

Con base en el análisis de los datos se recomienda seguimiento permanente de las diferentes variables, al igual que monitoreo constante al nivel de satisfacción de los clientes.

\section{CONCLUSIONES}

El modelo Seis Sigma requiere un cambio en la mentalidad no solamente de la gerencia sino de toda la organización, orientándola hacia el mejoramiento de la confiabilidad interna -de los procesos- que se revierte en un incremento sostenido de la confianza externa -de los clientes y demás stakeholders-.

El mejor indicador de la calidad de una empresa es la confianza que genera en los clientes y demás stakeholders. Este incremento en la confianza se fundamenta en que las organizaciones que se imponen como meta lograr niveles de calidad Seis Sigma, en la práctica le apuntan a 3,4 errores por millón de oportunidades, lo cual es realmente muy bajo.

En las empresas colombianas hay una gran brecha por recorrer en cuanto a lograr difundir y aplicar adecuadamente Seis Sigma. La gestión de procesos productivos con base en los principios de $6 \sigma$ constituiría un avance en competitividad nacional.
Calidad es igual a "confianza". Un proceso de mala calidad es errático, costoso, inestable y no se puede predecir. La mala calidad es inversamente proporcional a la competitividad, a la confiabilidad de procesos y a la fidelización de clientes.

\section{REFERENCIAS}

Alonso, V. (2003). Six Sigma: Muy cerca de la perfección. Entrevista a Mikel Harry. WOBI. Gestión, 8, WOBI, World of Business Ideas. Recuperado en: http:// www.wobi.com/es/articles/six-sigma-muy-cercade-la-perfección.

Barba, E., Boix, F. y Cuatrecasas, L. (2000). Seis sigma: una iniciativa de calidad total. Barcelona, España: Ediciones Gestión.

Barbosa, E. (2012). Metodología para la integración de Seis Sigma y Lean en una empresa PyME: un enfoque participativo entre la academia $y$ las PyMEs Tamaulipecas. Universidad de León, México. Recuperado en: http://www.tdx.cat/handle/10803/113434

Bernal, C. A. (2010). Metodología de la Investigación. Bogotá, Colombia: Pearson.

Chase, R. (2012). Administración de operaciones: Producción y cadena de registros. México: McGrawHill. 
Introducción de la medida de calidad en la producción de aulas virtuales de la universidad EAN. Seis Sigma

Eckes, G. (2004). El six sigma para todos. Bogotá, Colombia: Grupo Editorial Norma.

Escalante, V. E. J. (2013). Seis-sigma: metodología y técnicas. México: Limusa; Noriega Editores.

Figuerola, N. (2009). Six Sigma. Recuperado en: http:// pmquality.files.wordpress.com/2009/08/seis-sigma-articulos.pdf

Gardner, D., Bryce, I., Kleinier, J., Pitt, B. (Pro.); Carnahan, M., Goddard, D., Lindelof, D., Straczynski, J. M., Brooks, M. (R.), and Forster, M. (Dir.). (2013). Guerra Mundial Z. Estados Unidos: Paramount Pictures; Skydance Productions; Plan B Entertainment. United International Pictures (UIP).

Gómez-Dacal, G. (2004). K sigma: teoría de las organizaciones y control de calidad (de la enseñanza). Salamanca, España: Ediciones Universidad de Salamanca.

Gutiérrez P., H. y De la Vara S., R. (2013). Control estadístico de calidad y seis sigma. México: McGraw Hill.

Kumar, D. (2009). Six sigmas, las mejores prácticas : una guía de la excelencia en el proceso de los negocios. Bogotá, Colombia: Panamericana Editorial: 3R Editores.

Motorola Museum of Electronics. (1997). Motorola Family Album. New York: Dick Young Productions. Recuperado en: http://youtu.be/oujbiQSBYjw
País Curto, J. R. (2013). BPM (Business Process Management): Cómo alcanzar la agilidad y eficiencia operacional a través de BPM y la empresa orientada a procesos. Recuperado en: BPMteca.com

Pande, P., Neuman, R. y Cavanagh, R. (2004) Las claves prácticas de Seis Sigma: una guía dirigida a los equipos de mejora de procesos. Trad. J.M. y E. Bernat de la primera edición en inglés de The Six Sigma Way. Team Fieldbook, 2002. Madrid: McGraw Hill / Interamericana de España. (Impreso en Colombia)

Pande, P. S., Neuman, R. P. y Cavanagh, R. R. (2002) Las claves de Seis Sigma: la implantación con éxito de una cultura que revoluciona el mundo empresarial. Trad. Ana Matos de la edición original en lengua inglesa (2000) Madrid, España: McGraw Hill / Interamericana de España.

Pande, P. S. y Holpp, L. (2002). ¿Qué es seis sigma? Madrid, España: McGraw-Hill.

Pande, P., Neuman, R. y Cavanagh, R. (2000). The Six Sigma Way: How GE, Motorola, and Other Top Companies are Honing Their Performance. McGraw Hill Professional. Recuperado En: http://books.google.com.co/ books?id=ybOuvzvcqTAC\&printsec=frontcover

Ríos, G. y Ricardo, M. (2013). Seguimiento, medición, análisis y mejora en los sistemas de gestión: enfoque bajo indicadores de gestión y balanced scorecard. Bogotá, Colombia: ICONTEC. 
Schroeder, R. G.; Meyer Golstein, S. y Rungtusanatham, M. (2011). Administración de operaciones: conceptos y casos contemporáneos. México: McGraw-Hill Interamericana.

Thomke, S. H. y Mona, S. ( 2013). The Dabbawala System: On-Time Delivery, Every Time. Harvard Business School Case 610-059. Recuperado en: http://www. hbs.edu/faculty/Pages/item. aspx?num =38410

Valderrey Sanz, P. (2013). Herramientas para la calidad total. Madrid, España: Starbook.
Ventura, G. (2014). Un nuevo enfoque de la confiabilidad, usando el seis sigma en la matriz de riesgo. Recuperado en: http://cidesi.com/contenidos2014/ confiabilidad.pdf

Vitasek, K., Manrodt, K. y Kling, J. (2013) Vested: el modelo que $P \& G$, Microsoft y McDonald's adoptaron para redefinir el éxito en los negocios. Bogotá, Colombia: Norma.

Wheat, B. (2008). Seis sigma: una parábola sobre el camino hacia la excelencia y una empresa esbelta. Bogotá, Colombia: Grupo Editorial Norma, 2008.

Wobi World of Business Ideas (2012). Cinco preguntas a Jack Welch. en: http://www.wobi.com/es/wbftv/ cinco-preguntas-jack-welch 